\title{
COMPUTERS IN
} INDUSTRY

\section{Building holarchies for concurrent manufacturing planning and control in EtoPlan}

\author{
M.M.T. Giebels ${ }^{\mathrm{a}, *}$, H.J.J. Kals ${ }^{\mathrm{a}}$, W.H.M. Zijm ${ }^{\mathrm{b}}$ \\ ${ }^{\mathrm{a}}$ Laboratory of Design, Production and Management, University of Twente, P.O. Box 217, AE Enschede, The Netherlands \\ ${ }^{\mathrm{b}}$ Stochastic Operations Research, University of Twente, AE Enschede, The Netherlands
}

\begin{abstract}
The increasing versatility in order characteristics calls for planning and control systems that are able to evolve in time. Traditional hierarchical systems are usually based on a function-oriented static control structure in which all orders and products are handled similarly. Due to the dynamically changing characteristics of manufacturing environments these static control structures are not suitable anymore. Hence, a concept description and a prototype implementation for concurrent manufacturing planning and control (EtoPlan) based on multiple and temporary hierarchies (holarchies) are presented. The alternative control structure allows among other things to bridge the gap between process planning and production planning. The EtoPlan order planning method explicitly models the uncertainty in the information due to incompleteness of process planning information and shop floor randomness. (C) 2001 Elsevier Science B.V. All rights reserved.
\end{abstract}

Keywords: Flexible control; Integration; Holonic control; Stochastic planning

\section{Introduction}

An essential demand for future manufacturing control systems is the ability to deal with the increased complexity due to a higher product variety, smaller batches and shorter throughput times. Hence, it is generally recognised that future manufacturing planning and control systems will become more flexible and integrative compared to the presently existing systems. The versatility in order characteristics calls for control systems that are able to evolve in time. There is a need for continual restructuring the control architectures used within manufacturing companies.

\footnotetext{
* Corresponding author. Tel.: +31-5348-92520; fax: +31-5348-93631.

E-mail address: m.m.t.giebels@wb.utwente.nl (M.M.T. Giebels).
}

Today, the hierarchical control structures applied in most manufacturing control systems are rigidly modelled in the system implementation phase, which results in static structures for controlling the manufacturing planning activities. These systems are, therefore, too rigid for handling the dynamically changing situation in the companies. Hence, more and more researchers apply socio-technical methods in order to be able to integrate evolution in manufacturing control systems. Examples include the fractal factory [1], biological manufacturing systems [2], and holonic control systems [3].

Although many researchers recognise the importance of these concepts for future manufacturing systems, few have already succeeded in implementing evolutionary systems that can satisfactorily be applied in practice. Unfortunately, implementations of evolutionary control concepts are often not as flexible as 
presumed and initially pursued. Possible causes include the use of a limited set of rigid planning rules, and the need to freeze (crystallise) a control structure for reasons of applicability. It is a challenge to build a system able both to handle dynamic production rules and to reorganise itself continually according to changing requirements, constraints and demands.

In this article, we present an holonic evolutionary planning and control system based on building multiple and temporary hierarchies. The system, named EtoPlan (engineer-to-order planning), has been developed for concurrently controlling the technological and logistic planning tasks in make- or engineer-toorder manufacturing environments. The system is capable of dealing with large amounts of uncertainty, caused by incomplete and unreliable information. Concurrent planning methods that can deal with uncertainty are particularly needed for higher-level planning of the specified type of manufacturing environment. The already available technological and logistic information is presented to the various planners (salesmen, process planners, and production planners) through suitable views. Important is what information must be presented and how [4]. Each planning activity requires its own representation of the information that is available at the various locations in the company. The system processes the information, tailored to the needs of the planning activity that is being performed. For instance, the system can provide the process planner with information about the expected availability of the resources that are taken into consideration for executing a given process step.

In the next section, the characteristics of hierarchical control are discussed. Section 3 elaborates the differences between hierarchical control and the holonic control concept. In this section, we conclude that hierarchies are indispensable for controlling a dynamically changing complex manufacturing environment. A hierarchical control structure, however, can only deal with such dynamic environments if it is continually adapted to changing circumstances. The EtoPlan concept, presented in Section 5, has been developed for meeting these demands. A specific Information Management concept, which is briefly discussed in Section 4, serves as the backbone for the EtoPlan concept. In Section 6, a part of the EtoPlan prototype implementation is described in order to show the practical applicability of the concept.

\section{Characteristics of hierarchical control}

The term hierarchical control needs a clear definition due to the fact that completely different meanings are being used in literature. In the ISO 4258 International Standard (concepts and rules for enterprise models), the concept of hierarchy is defined as follows [5]:

Hierarchy is a principle by which real world items and abstractions can be ranked and ordered. There are two kinds of hierarchies: part-of hierarchies and kind-of hierarchies. Part-of hierarchies represent the composition of elements or the decomposition of systems. Kind-of hierarchies represent levels of abstraction that are ordered by generalization and specialization.

In manufacturing control research often references are made to the three principles of hierarchical control described by Albus for a hierarchical robot control system. These principles are [6,7]:

- Different levels are introduced to enable the decomposition of problems in order to reduce the complexity and to limit the responsibility and authority at each level.

- Each level has a distinct planning horizon, the length of which decreases when going down the hierarchy.

- Control resides at the lowest possible level.

Another important characteristic of hierarchical manufacturing control systems refers to an increased level of detail in the plans when descending within the hierarchy. Hierarchical control makes it possible to perform aggregate planning with incomplete information on the higher control levels.

A distinction is also made between proper and modified hierarchical control. In the so-called proper hierarchical form, there are no control flows between the control entities on the same hierarchical level. Modified hierarchical control allows some co-ordination between these control entities, without having to consult a higher-level control entity. This modification decreases the chance of overloading the higher-level control entities, a risk that was already noticed by Galbraith in the early seventies [8]. On the other hand, the control entities, then, need information about the other entities in the system and a co-ordination protocol must be developed. It is, therefore, questioned whether an overload is more likely to occur in proper 
hierarchical control systems than in modified hierarchical control systems.

The above-mentioned characteristics of hierarchical control are not always clearly stated by researchers in the field of manufacturing control. Some misconceptions are often formulated, particularly in comparison analysis with heterarchical and holonic control systems.

Firstly, hierarchical control has often been misinterpreted as (nearly) equal to centralised control by some researchers. It is, then, assumed that only two hierarchical levels exist, where the entities on the lowest level function as 'slaves' and all planning activities are performed by one central planner, the 'master'. In this way, no effect is acquired with regard to the decomposition of the planning task and, thus, the planning problem as was the main reason for development of hierarchical control in the first place. The notion of hierarchical control as master-slaves relations, can only be hold if hierarchical control is assumed to be equal with centralised control. Centralised control is, however, in no way in conformity with the principles of hierarchical control as defined by Albus. A hierarchy with more than one hierarchical planning and control level will always be based on the distribution of the planning task, and can, therefore, never exist of one central planner (master) and slaves with no planning functionality.

The following example, based on the modified hierarchical FACT concept developed by Arentsen [9], shows that the before mentioned assumption is wrong. The highest level (factory control) performs the capacity planning task considering a time horizon of approximately 3 months in an average job-shop. Cell control performs the detailed scheduling task $( \pm 2$ weeks), workstation control performs the job sequencing task ( \pm 1 day) and equipment control is responsible for controlling the production activities. Consequently, no central production plan is drawn up, but multiple plans are generated in parallel by various planners on multiple levels of abstraction.

Somewhat related to this master-slave misconception is the assumption that lower level control entities are subordinate to the higher-level control entities in hierarchical control. Although the decision-making responsibilities are often determined on the basis of the hierarchical control levels, this is not a necessity in hierarchical control systems. Van Aken states that organisational structures usually involve a combination of stratification and hierarchy $[10,11]$. These features should, however, not be confused. Hierarchy is characterised by problem decomposition (in order to deal with complexity) and aggregation levels in the planning (in order to deal with incomplete information). Stratification refers to the order of control entities with regard to conflict solving between entities' interests.

Another misconception regards the possibility of replanning when disturbances occur. Within a hierarchical control system, the problems are dealt with on the lowest possible level. That means, bottom-up. If, for example, a machine breakdown of short duration has occurred, it will probably not significantly disrupt the schedule as has been developed on cell level. Such a problem is recognised by equipment control and solved on workstation level by the workstation controller. Problems are only reported to the next higher control level if the problem is so drastic that the subordinate level cannot meet the demands received from the higher-level control module. In other words, the problem-solving autonomy of the control entities is limited if the problem also negatively influences the progress of the production plans on the higher control levels.

It is often stated that a negative feature of hierarchical control systems is the inability to deal with regularly occurring disturbances, e.g. by Bongaerts et al. [12]. This is unquestionably true if the aforementioned centralised interpretation of 'hierarchical control' is assumed, as is done by Bongaerts et al. in their tests from which they draw this conclusion, viz. a detailed plan is centrally drawn up and executed by 'slaves' without the possibility of re-planning in realtime. Most hierarchical control systems they refer to, a.o. the FACT system [9], are, however, implementations of a more intelligent (real) hierarchical concept. Real hierarchical control systems are modelled according to the decomposition and aggregation principles of hierarchical control, which makes effective disturbance handling possible.

\section{The differences between hierarchical and holonic control}

Holonic control is often described as a concept that combines the best features from both hierarchical and heterarchical control [12]. 
The idea of holonic manufacturing is based on the concept of 'holonic systems', developed by Koestler [13]. Koestler defines a holarchy as "a hierarchy of self-regulating control building blocks (holons), which function (a) as autonomous wholes in supraordination to their parts, (b) as dependent parts in subordination to controls on higher-levels, (c) in coordination with their local environment" [14].

Considering the description of hierarchical control in the previous section, the following question comes naturally: are there fundamental differences between hierarchical control systems and holonic control systems?

The answer is yes, there are. According to Bongaerts et al. [12], holonic control differs from traditional hierarchical control on the following aspects:

- Holons can belong to multiple hierarchies.

- Holons can form temporary hierarchies.

- Holons do not rely on the proper operation of each holon in the hierarchy to get their work done.

The latter of the three differences refers to the increased autonomy of the holons compared to the control modules in hierarchical control. The work plans that are provided to the subordinate holons are considered as an advice. Furthermore, production rules are applied in order to prevent the natural behaviour of aiming at local optimisation by the holons. Unfortunately, this modification potentially introduces unreliability in the system. Subordinate holons are not aware of the arguments that have resulted in the actual work plan drawn up by the higher-level holon. It is, therefore, possible that ineffective decisions are taken by the subordinate holons that disrupt the execution of some work plans dispatched to the other subordinate holons. Therefore, applying abstraction planning, as described by Washington [15], may prove to be a more suitable approach. Abstraction planning means that the higherlevel holons do define rough goals, requirements and constraints only. The question how to meet these goals and requirements is left to the subordinate holons. These holons define the more detailed goals, requirements and constraints themselves, which, in turn, are handed over to their lower level holons, etc. A subordinate holon reports to the higher-level holon(s) if it is not able to meet the specified requirements, e.g. due to occurring disturbances. Subsequently, the higher-level holon initiates (roughly defined) corrective actions if necessary. The above-mentioned control rule that is necessary for integrating abstraction planning in manufacturing control, complies with the definition of an invariant in holonic manufacturing systems [16]. According to Bongaerts et al. an invariant is a logical proposition that expresses the requirements on the behaviour of a holonic control algorithm. Bongaerts applies the strategy of increasing the autonomy for the subordinates by considering the (detailed) work plans obtained from the higher control level as an advice. In contrast, we increase the autonomy of the subordinates by leaving the detailed planning decisions to the subordinates, while simultaneously demanding that the roughly described requirements should be met.

The other two differences between holonic and hierarchical control, namely multiple and temporary hierarchies, as mentioned by Bongaerts et al. [12], are believed to be essential characteristics for future manufacturing systems.

Multiple hierarchies are necessary for achieving concurrency in the various manufacturing planning processes. Applying an object-oriented approach based on the holonic principles of simultaneously being a 'whole' and 'part' of other 'wholes' [13] may lead to a final demolishment of the walls that still exist between, e.g. process planning and production planning departments. According to Valckenaers et al. [17], each hierarchy can reflect a view on the system, e.g. a scheduling view, a process planning view, a maintenance view, etc. A specific concept on Information Management that is currently developed in the Laboratory of Design, Production and Management at the University of Twente [4], serves as a backbone for creating multiple views in multiple domains of the order, resource and product information structures (see Section 4).

Temporary hierarchies can solve the problem of increased dynamics in manufacturing companies. Traditional hierarchical systems are based on a functionoriented static control structure in which all orders and products are handled similarly. Due to the changing characteristics of the manufacturing environments (viz. high product variety, smaller batches, shorter throughput times), these static control structures are not suitable anymore. There is a need for continually re-arranging the control structure of the company, 
according to the dynamically changing situation in the factory. This especially holds for engineer-to-order and make-to-order manufacturing environments.

\subsection{The need for flexible hierarchies}

The main advantage of heterarchical control compared to hierarchical control regards to the flexibility of re-arranging relations between the objects in the system. Hierarchical control is characterised by a static structure of control. Heterarchical control systems are not structured and are, therefore, potentially more capable of dealing with complex ever-changing manufacturing environments. If a manufacturing environment is fairly static, a hierarchical control system is suitable for controlling the orders and the resources. However, for controlling a dynamic manufacturing environment - characterised by small batches, a high product variety and many orders that are to be controlled in parallel - it is not adequate to apply a static hierarchical control system. Heterarchical control systems require, on the other hand, simple and sound control rules in order to regulate the local planning processes. One may doubt whether it is possible at all to define sound rules, because of the variety of production goals and the complexity of the environment. For instance, dispatching rules like Earliest Due Date (EDD) often tend to yield a low performance in complex manufacturing environments, due to the absence of an integrated view. More intelligent heuristics, e.g. the shifting bottleneck scheduling approach, often outperform these dispatching rules in practice [18]. Such heuristics however are not applicable in heterarchical systems due to the philosophy of not allowing higher-level planning and control methods.

As discussed in Section 2, hierarchies can also be useful in dealing with disturbances. In hierarchical control systems, disturbances can be dealt with at the most suitable hierarchical control level. Minor problems are solved on a low hierarchical level. Major problems - which propagate through a large part of the production plans - are dealt with on a higher hierarchical level in order to minimise the negative impact of such major disturbances on the global goals of the company.

In conclusion, we can state that hierarchies are indispensable for controlling a dynamically changing complex manufacturing environment. A hierarchical control structure, however, can only deal with such dynamic environments if it is continually adapted to changing circumstances.

\section{Information Management}

The EtoPlan concept has been developed for manufacturing planning and control in engineer-to-order environments. In engineer-to-order environments, there is an evident need for planning methods that can perform the traditionally separated planning processes (e.g. process planning and production planning) more concurrently. There is, however, a fundamental difference between the information processing approach traditionally applied for either process planning and production planning. Process planning is normally structured in an order-oriented way, whereas production planning is structured in a resourceoriented way.

In process planning, a technological plan for realising the product ordered by the customer is generated applying several levels of abstraction. First, the methods required for producing the product are selected. Second, the set-ups are determined and, third, the tool paths are calculated. Traditionally, these planning steps are performed without any concern of capacity problems that might occur due to other orders with which resource capacity must be shared. Even the resource allocation decision is, often, not taken into account in order to leave ample flexibility in decisionmaking to the production planning department. Concluding, process planning is typically performed in an order-oriented manner.

On the other hand, production planning and control is, traditionally, structured in a resource-oriented manner. A factory controller considers all available resources at the factory level. A cell controller controls the subordinate departments and the workstations in the department are controlled by a workstation controller.

Thus, a static hierarchical resource structure constitutes the usual basis for planning and controlling the logistic aspects of the orders, while a hierarchical order structure forms the basis for the planning of the technological aspects. The mismatch between these structures makes it almost impossible to perform 
these tasks simultaneously. Many researchers have tried to integrate the logistical and technological planning functions by defining interfaces between the two planning functions on several levels of aggregation, a.o. Cho et al. [19] and Huang et al. [20]. A significant drawback of the interfacing approach is the rigidity that is created due to the necessity of establishing hierarchies in both process and production planning that, in a way, are uniform for communication purposes between the two hierarchies. To avoid this drawback, the levels of abstraction required for either production planning and process planning must be structured similarly.

This is conceptually impossible as long as the mentioned differences between the hierarchical structure of process planning (order-dependent) and production planning (resource-dependent) continue to exist. It is practically impossible to build static hierarchies in both an order-dependent and a resourcedependent way. For that reason, building temporary hierarchies seems to constitute a more natural way for achieving concurrency in manufacturing planning. In Section 5, it is described how temporary hierarchies are modelled in the EtoPlan concept.

The functional planning departments (or expert groups) in a company all make their own decisions in order to meet the requirements and constraints of the company and its environment. These departments deal with the same order product and available resources, but all in their own specific domain. However, most decisions taken by a department are also of concern for other departments, because these decisions limit the solution space of successive decisions. Aiming at concurrent manufacturing engineering will increase the need for feedback and inter-departmental communication, which can lead to extremely complex and uncontrollable flows of information between the separate departments [24]. An additional problem is the difficulty of backward transformation of information through the planning stages [4]. For instance, it is impossible to transform manufacturing instructions drawn up by the planning department into the original detailed design specification.

Mainly for these reasons, a new concept for Information Management is proposed by Lutters and coworkers [25]. The information required for making decisions is 'pulled' to the departments instead of 'pushed' from one department to the next in the

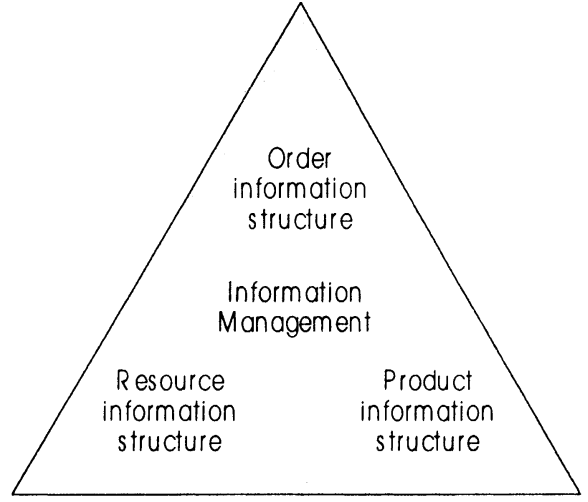

Fig. 1. The three information structures as part of Information Management [24].

planning cycle as is the case in traditional manufacturing planning. Central in this approach is the "need for information' which drives the control and the course of the manufacturing planning processes.

All information generated by the separate departments is attached to a general model containing the following three information structures (Fig. 1):

- product information structure (PRIS);

- order information structure (OIS);

- resource information structure (RIS).

The three information structures contain all information generated during the whole life cycle of the products, orders, and resources, respectively. These classes of objects are regarded as the basic elements present in a manufacturing environment. All the information can be stored in these three information structures or as attributes attached to relations between these structures.

Hierarchies of the objects are built up during their life cycle based on the fundamental structure. This is generically done, independent of the type of object (e.g. operator, design feature, or production activity) the information bears reference to. No pre-defined hierarchies exist in the generic information structures. The information structures evolve in time regarding both the hierarchies and the values of the attributes.

Which planning processes are performed and in which sequence depends on the need for information initiated by external or internal events that subsequently initiate the planning processes for filling up the information required for decision-making in order 
to deal with the changed situation. This approach differs fundamentally with the traditional scenariobased planning strategies, where a pre-defined sequence of planning activities has to be performed. In elaborating this concept for the order information structure, the order life cycle (from the initial offer until delivery to the client) is oriented upon the order instead of on, e.g. the scheduling process.

\section{A concept based on building manufacturing holarchies}

In the previous section, it has stated that the planning hierarchies used for process planning and production planning and control must be similar. This can only be achieved if the hierarchies themselves are adaptable. As a result, the system architecture proposed in this section is based on building temporary hierarchies for manufacturing planning and control. The concept for dynamic manufacturing planning and control, named EtoPlan (engineer-to-order planning) has been developed in the Laboratory of Design, Production and Management at the University of Twente. The EtoPlan concept is based on the generic Information Management concept described in the previous section. The EtoPlan concept consists of an order planning methodology for resource loading and a system architecture by which all planning functions and an evolutionary control structure are defined.

\subsection{System architecture}

As was concluded earlier, there is a need for flexibly building temporary hierarchies of manufacturing resources in order to be able to handle the diversity in characteristics of the orders that are executed simultaneously. In this section, the structural model of EtoPlan is presented. The structural model is part of the system architecture of EtoPlan. It describes the structure of the control entities and the relations between them.

In the EtoPlan concept, temporary hierarchies are built by dynamically grouping the resources according to the requirements of individual orders to be planned. In this way, many different kinds of products can be manufactured simultaneously on the shop floor, while still being able to consider the multilateral influences of the orders. This is an important feature for small batch manufacturing, where the routings of the diverse production orders may vary highly. Although the routings and accompanying aggregations of resources for manufacturing the products will differ significantly, the diverse orders may claim the same physical resources in the same period of time. Then, capacity requirement conflicts may occur between these orders. The temporary hierarchies of resources belonging to individual orders are, therefore, not independent of each other.

The multiple and temporary hierarchies (holarchies) of resources are built up as follows.

For every individual (sub)order, a unique group of applicable resources, is drawn up. A resource is considered applicable if

- the resource is considered capable of meeting the already known technological requirements in the roughly defined process plans for executing (a part of) the order;

- the resource is considered to be available during a time period that is roughly planned for executing (a part of) the order.

An Applicability Group (AG) is a virtual group of resources that are considered applicable for the (partial) execution of a definite order.

In the above definition, the word virtual refers to the fact that the configuration of an AG does not imply physical grouping of resources, e.g. the case in group technology. A resource can be anything, e.g. a machine tool, a tool, a fixture, material, an operator, etc. Only the resources that are relevant for the planning and control decisions performed on the aggregation level of a certain order are grouped in the accompanying AG. On the higher-levels of aggregation mainly machine tools will be considered. For some specific production orders, it can, however, be recommended or even essential to take other kinds of resources into account, e.g. in many make- or engineer-to-order companies, machine utilisation rates are relatively low and the capacity constraints mainly depend on the operators available on the shop floor. In that case, it is necessary to include the planning of operators into the capacity planning task, which makes it necessary to determine what and how many operators should be allocated to the AGs. 
When more detail is included in the manufacturing plans, i.e. on the lower levels of abstraction, also the need for including more types of resources (e.g. tools) in the plans increases. These resources will then be added to the AGs. This will make the planning of for instance tool paths in micro-process planning or the logistic planning of auxiliary jobs (e.g. tool assembly) possible.

For example, if a special group of operations can only be executed on a dedicated machine tool with pre-assembled cutting tools and fixtures, it is then likely that an AG consisting of the aforementioned resources and two AGs for performing the auxiliary jobs (tool assembly and fixture mounting) will be drawn up by the planners. Such a situation is shown in Fig. 2. Two different operators are also part of the AGs for performing the operations; an operator for controlling the CNC-milling machine tool (operator 1, suborder 2.3) and an operator for performing the tools and fixture mounting activities (operator 2, suborder 2.1 and suborder 2.2). A process planner is also added to the AG belonging to suborder 2.3, because the NCprogram for the CNC machine tool has not yet been defined.

The AGs exist throughout the life cycle of the order they are created for. An AG is initiated by either a higher-level AG or a newly entered order. It exists until all lower level AGs are triggered or until the related jobs are completed. Depending on the complexity of the product to be produced, the number of hierarchical levels in the order structure will differ for different orders. In this way, the holonic structure consisting of temporary and multiple hierarchies of resources is created.

Although the AGs are directly related to the life cycle of the orders, the AGs will be dealt with by the resource information structure (see Section 4). Resources are part of multiple AGs on multiple levels of aggregation as there are numerous orders being planned and executed simultaneously as is very likely in a make- or engineer-to-order small batch manufacturing environment. For example, a resource can simultaneously be part of (a) a small AG connected to a job which is presently executed on a workstation and (b) a large AG connected to a production order for which only the macro-process planning has been performed and a detailed routing has not yet been generated. In practice, a resource can be part of up to hundreds of AGs simultaneously.

The EtoPlan concept aims to take into account both the optimisation of global goals in production and the solution of short-term production problems. The solution of global planning problems normally covers a relative long time period, whereas detailed operational activities concern a significantly shorter time period. This is however not always the case. Some planning activities already require considerable detail in an early planning stage. For example, when the production of a non-modular fixture for a certain operation can only be performed outside the company, then the detailed fixture design and, thus, the detailed process planning should be performed in an early stage. The levels of detail and the time of planning largely depend on the orders considered. Therefore,

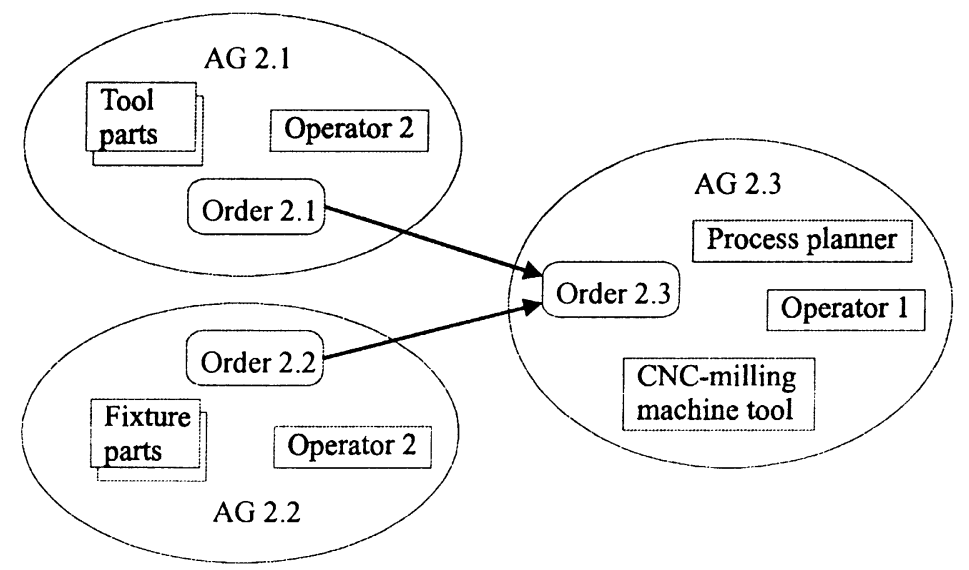

Fig. 2. An example of Applicability Groups. 
the abstraction levels in EtoPlan are determined in an order-oriented way.

In summary, no static hierarchies of resources are defined, but for every production order a temporary hierarchy (holarchy) of resources is created. This temporary hierarchy of resources (AGs) corresponds to the hierarchy of the order structure. The manufacturing planning and control functions are performed by the controller of the AGs.

Every AG in the hierarchy, and thus, every order step, is controlled by an accompanying autonomous AG controller.

When an AG is instantiated from a higher-level AG, the responsibility for planning the activities to be performed by the new AG in a specific time period is shifted on to the AG controller belonging to this new AG. The planning of the activities - technological as well as logistic - is then performed by the planning function of that AG controller. Fig. 3 schematically shows the overlap in time periods considered by the AG controllers on different aggregation levels. The dark Gantt bars represent the orders that are already created from a higher-level order by the AG controller of the AG belonging to the higher-level order. The lighter Gantt bars are already planned, but not yet dispatched by the higher-level AG controller, which means that no AG controller for these AGs has been initiated yet. Until an autonomous AG controller is initiated for such an order, all planning activities considering this specific order are still performed by the higher-level AG (order) controller.

\subsection{Integrated order planning methodology}

The EtoPlan order planning methodology supports the integration of the company management function 'order acceptance' and the higher-level planning tasks in both process planning (i.e. macro-process planning) and production planning (i.e. resource loading). Macro-process planning concerns the rough technological planning of aggregate production activities, such as the rough determination of the routing of orders through the factory. Preferably, resource loading is performed concurrently with the macro-process planning process. In this way, the decisions to be taken can be tuned to one another.

The goals of the EtoPlan decision support concept for aggregate order planning are:

- providing a reliable view on the availability of the resources and the resource groups (e.g. cells or workstations);

- supporting the aggregate planning of the production activities with regard to start times, lead times, and variable costs;

- decision support for the order acceptance process concerning:

$\circ$ due date setting and feasibility analysis;

o the estimation of variable costs that result from critical situations in production planning.

The focus of the EtoPlan aggregate order planning methodology is on providing resource loading views for all (aggregate) production activities that are specified by the macro-process planning process. In this way, it becomes possible to support the macro-process planning task with logistic consequences of technological decisions. Due to the significant incompleteness of the technological plans at the macro-process planning stage, one has to deal with uncertainty in the information concerning processing times, required resources and routings. In other words, the input information for planning the orders in time cannot be considered deterministically. Therefore, the input data is modelled by means of beta-distributions for the processing times, and temporary groups of resources (AGs) with attached chances of allocation. Next, the

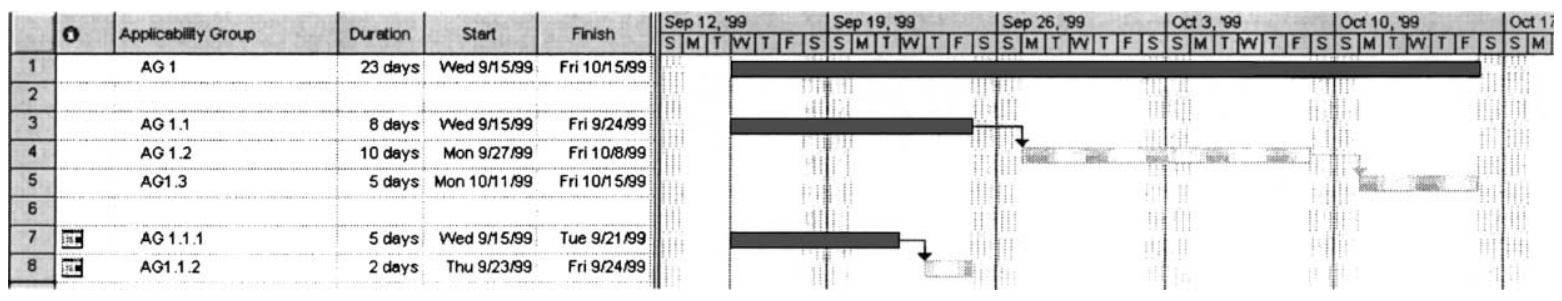

Fig. 3. Time overlap within an order hierarchy. 


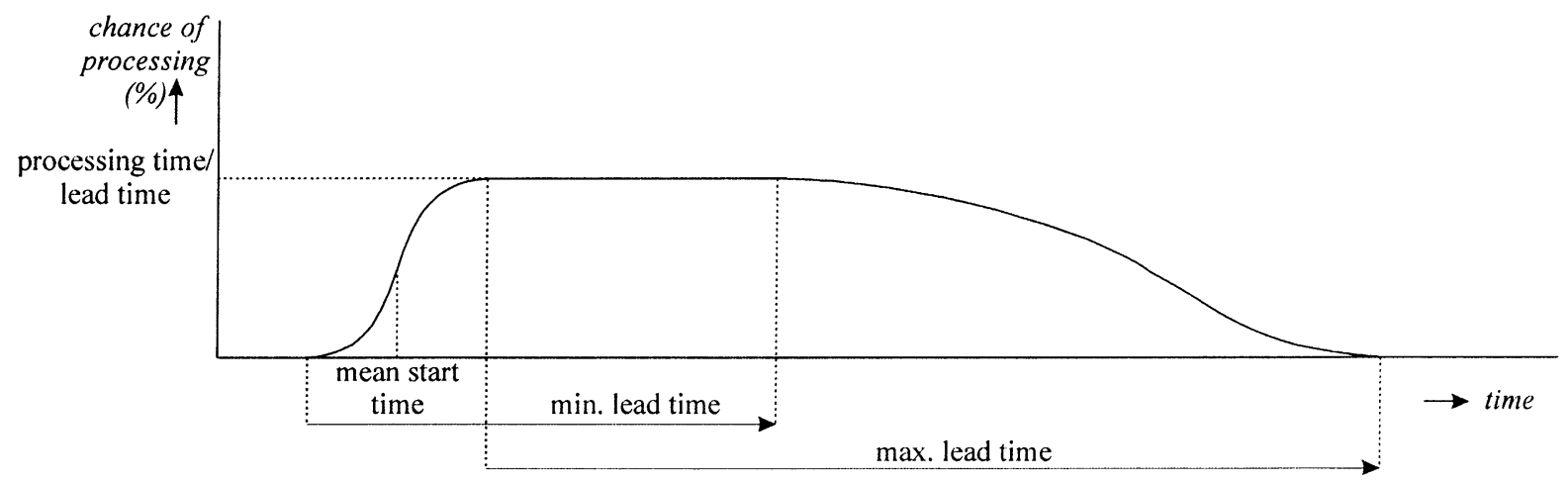

Fig. 4. Generic order profile.

production planner plans the lead time and start time of a production activity based upon a beta-distribution and a normal distribution, respectively. A more detailed explanation about both the stochastic modelling techniques and the way these stochastic values are applied in the integrated planning methodology has been described by Giebels et al. [21].

Because of the use of distributions of lead times and starting times instead of deterministic values in the EtoPlan order planning concept, a new representation form for the activities had to be developed. This resulted in the development of a generic order profile as depicted in Fig. 4. The shape of the probabilistic order profile results from combining the beta-distribution of the lead time, the normal deviation of the start time, and the mean processing time. The profile represents the order's chance (percentage) of actually being processed at that moment in time. The area under the order profile corresponds to the required processing time, which makes it possible to evaluate the utilisation of the resources required for executing the order. Consequently, the profile becomes smaller in the vertical direction in proportion to the relation processing time/lead time. For example, let the lead time be $10 \mathrm{~h}$ and the processing time $2 \mathrm{~h}$. The height of the order profile can then maximally reach the $20 \%$ chance of being executed at that moment in time. If all variances of the distributions are set to zero and the processing time equals the lead time, then the order profile corresponds to a rectangle as used in normal Gantt charts.

In make- or engineer-to-order manufacturing, the order planning decisions highly depend on the type of orders the decisions refer to. For instance, the solution approach for dealing with a (sub)order that most probably will exceed its due date will depend on answers to questions like:

- Does the due date violation also imply exceeding an already accepted delivery date?

- Can we postpone the delivery dates and to what costs?

- Can we still increase the priority of this order so as to decrease the lead time?

- Are there possibilities to shorten the processing times or to reduce the number of process steps by adjusting the process plans?

- Can we decrease the lead times by batch splitting and parallel processing?

It is clear that a large number of solutions are potentially available. Therefore, the process of solving problems in resource loading is rather versatile and typically depends on the type of orders one has to deal with. For this reason, a classification of order characteristics for resource loading in make- or engineerto-order manufacturing has been suggested [22]. More research is currently performed on developing intelligent decision support tools in EtoPlan based on the order classification results.

\section{The EtoPlan prototype software implementation}

A prototype decision support system for integrated order planning (EtoPlan) has been developed. Its purpose is to analyse and test the practical applicability of the system architecture and the accompanying concept 
for integrated order planning. The implementation covers the building of the AG hierarchies (holarchies) and the planning on the higher aggregation levels, where both macro-process planning and capacity planning take place. The EtoPlan system provides the user with information about resource availability estimates with regard to individual resources and the Applicability Groups. It also reports about capacity problems that are expected in the near future and it provides insight in the consequences of the current capacity planning decisions for the additional costs. Subsequently, a human planner can, possibly with the help of other planning tools, take the decisions necessary for meeting the company's goals. In particular, EtoPlan supports the planner in determining start times, processing times, lead times, Applicability Groups, due dates, suborders, costs, and overtime work.

The EtoPlan system has been developed using Microsoft Visual $\mathrm{C}^{++}$Developer Studio 6.0 [23]. The system structure is dynamically build up in an object-oriented way by continually creating, deleting and changing order structures and Applicability Groups.

An AG with two milling machine tools (MT002 and MT007) has been created for this order. The maximum capacity of this group of resources is $15 \mathrm{~h}$ per day. The estimated availability $(13 \mathrm{~h})$ represents the maximum capacity minus an estimation of the idle times of the resources due to the unavailability of other resources required for executing the (sub)orders. The order profile drawn on the $x$-axis, represents the estimated capacity requirement for executing order 100.010.010. The order profile is derived from the generic order profile, depicted in Fig. 4.

The thick line in Fig. 5 represent the estimated loading profile of the group of resources (AG) that corresponds to order 100.010.010. This line is drawn up by putting together the order profiles of all the orders that need one or more resources (i.e. are part of their AGs) that belong to the AG considered - in this case, the AG of order 100.010.010. The area under the line between two dates (e.g. area $\mathrm{A}$ in the figure) corresponds to the estimation of the required total processing time at the resources that belong to the $\mathrm{AG}$ during a certain time period.

The thin line shows the loading profile if the estimations of the maximum lead times are considered instead of the beta-distribution of the lead time. The latter will probably happen during the time periods with a high work in process (WIP). A peak in the thick line indicates that there is a high probability that a capacity problem will occur at that moment in time. This will go hand in hand with a high WIP. As a result, the lead time of most suborders involved will increase. As expected, the thin line (maximum lead times) shows a smoothing effect compared to the thick line (lead time distribution). If peaks in the thick line occur and no sufficient corrective actions can be taken, it is

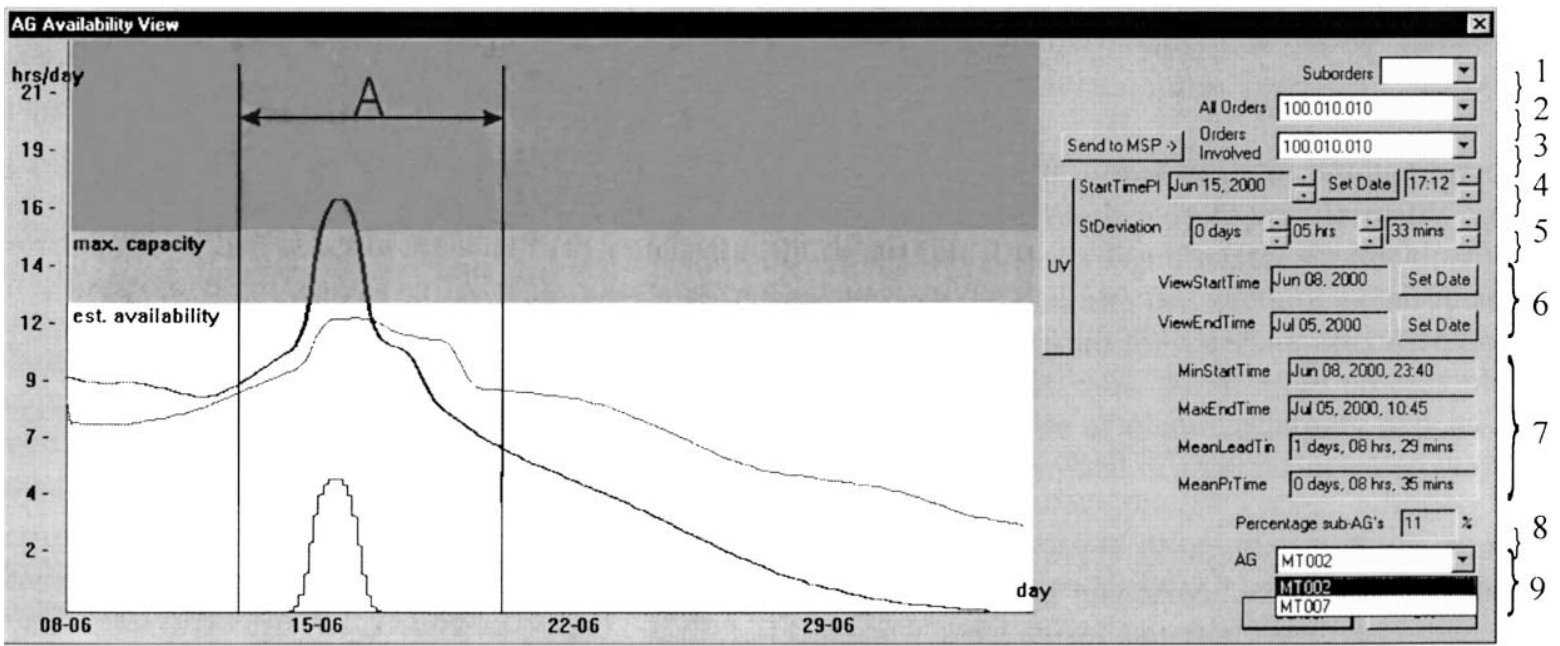

Fig. 5. An example of an Applicability Group availability view. 
likely that the thin line represents the actual situation that will arise.

The list boxes and the input buttons on the right side of the graphical user interface (GUI), as shown in Fig. 5, are used for planning some values of the (sub)order belonging to the Applicability Group considered. The control boxes indicated with a number in the figure are explained:

1. A combo box with the suborders of the order considered that are already initiated.

2. All (sub)orders in the system. The selected order (100.010.010) is the order that is currently being planned.

3. A combo box with all (sub)orders that influence the loading profiles, i.e. all (sub)orders which share one or more of the resources of the AG and which are to be executed, in this example, between 8 June and 5 July. If the user indicates a smaller time period (e.g. period A), then the orders involved in this time period are shown in this combo box. This can be useful if the planner wants to be informed about which orders are critical, and therefore, cause peaks in the loading profiles.

4. Used for start time/date determination.

5. Used for the determination of the standard deviation over the start time.

6. Setting the range of the time period that is shown on the $x$-axis.

7. Some information about the order for supporting the planner in making the planning decisions.

8. The percentage of the processing time of the order which has been planned already in more detail by means of its suborders.

9. The resources contained in the Applicability Group that belong to the order considered.

A more extensive description of the EtoPlan prototype implementation has been presented by Giebels et al. [21].

The prototype implementation has shown that it is actually possible to build temporary hierarchies during the actual planning processes, i.e. when the EtoPlan system is running [21]. The resulting flexibility facilitates an order-oriented approach which makes it possible to handle a high variety of orders with regard to characteristics such as product uniqueness, hierarchical levels, lead times, and so on.
In order to integrate intelligent navigation support in the system, additional research is currently performed. Such a navigation tool will support the user (process planners and production planners) in achieving the planning goals mainly by showing the required route for decision-making.

\section{Conclusions}

The differences between the hierarchical and holonic control principles are analysed in this paper. Some supposed differences mentioned frequently in manufacturing control literature do, in fact, refer to centralised control instead of hierarchical control and are, therefore, classified as misconceptions. The main real difference between the hierarchical control and holonic control concepts regards the dynamics in the hierarchical structure. It is concluded that future manufacturing planning and control systems should apply temporary and multiple hierarchies (holarchies) in order to be able to flexibly control the activities in the manufacturing companies.

A concept (EtoPlan) has been developed for building such holarchies. In the EtoPlan concept, the temporary hierarchies are modelled by way of Applicability Groups (AGs) that consist of the resources that are considered applicable for executing a given (sub)order. In this way, for every order a unique hierarchy of resources is drawn up. By applying order-oriented and thereby multiple-hierarchies, it has become possible to bridge the information gap between process planning and production planning.

A specific Information Management concept serves as the backbone for this approach. The life cycles of the object classes products, resources and orders, are oriented on the information contained in the accompanying information structures, instead of on the processes concerned with this information. Which planning processes are performed and in which sequence depends on the need for information initiated by external or internal events that subsequently initiate the planning processes for filling up the information required for decision-making in order to deal with the changed situation. This approach differs fundamentally from the traditional scenariobased planning strategies, where a pre-defined sequence of planning activities has to be performed. 
The strategic choice, in the EtoPlan concept, of modelling the uncertainty due to incompleteness of process planning information and shop floor randomness enhances the reliability and, thereby, the feasibility of the production plans. An integrated order planning method has been presented briefly. It supports the integration of the company management function 'order acceptance' and the higher-level planning tasks in both process planning (i.e. macroprocess planning) and production planning (i.e. resource loading).

This EtoPlan concept has been implemented in a prototype software system. Presently, the system is tested under laboratory conditions. It can already be concluded that it is actually possible to build temporary hierarchies during the actual planning processes, i.e. when the EtoPlan system is running. The resulting flexibility facilitates an order-oriented approach which makes it possible to handle a high variety of orders with regard to characteristics such as product uniqueness, hierarchical levels, lead times, and so on.

Several goals are pursued for testing the system. First, it is examined whether the system adequately supports the human planners. Second, the influence on the throughput time and the reliability of meeting the due-dates is analysed. Third, it is expected that various performance indicators can be improved by a better co-ordination of the decision-making process. It is expected that the integration of a navigation tool in the EtoPlan prototype implementation will further enhance the value of the system for manufacturing planners.

\section{References}

[1] H.-J. Warnecke, The Fractal Company: A Revolution in Corporate Culture, Springer, Berlin, 1993; ISBN: 3-54056537-X.

[2] K. Ueda, J. Vaario, K.H. Ohkura, Modelling of biological manufacturing systems for dynamic reconfiguration, CIRP Annals 46 (1) (1997) 343-346; ISSN: 0007-8506,

[3] H. Van Brussel, P. Valckenaers, L. Bongaerts, J. Wyns, Architectural and system design issues in holonic manufacturing systems, in: Proceedings of the Pre-prints of the 3rd IFAC Workshop on Intelligent Manufacturing Systems (IMS'95), Bucharest, Romania, 1995, pp. 1-6.

[4] H.J.J. Kals, D. Lutters, The role of Information Management in intelligent manufacturing (invited keynote), in: Proceedings of the CIRP International Conference on Intelligent
Computation in Manufacturing Engineering (ICME'98), Capri, Italy, 1998.

[5] ISO, http://www.mel.nist.gov/sc5wn1/std-dft.htm, 1999

[6] J.S. Albus, A.J. Barbera, R.N. Nagel, Theory and practice of hierarchical control, in: Proceedings of the 23rd IEEE Computer Society International Conference, Washington, USA, 1981, pp. 18-39.

[7] A. Jones, A. Saleh, A multi-level/multi-layer architecture for intelligent shop floor control, International Journal of Computer Integrated Manufacturing 3 (1) (1990) 60-70.

[8] J. Galbraith, Designing Complex Organizations, AddisonWesley, Reading, MA, 1973; ISBN: 0-201-02559-0.

[9] A.L. Arentsen, A generic architecture for factory activity control, Ph.D. thesis, Faculty of Mechanical Engineering, University of Twente, Enschede, 1995.

[10] J.E. van Aken, On the control of complex industrial organizations, Ph.D. thesis, Eindhoven University of Technology, Martinus Nijhoff, Leiden, 1978; ISBN: 90-207-0840-6.

[11] A.J.R. Zwegers, On Systems Architecting — a study in shop floor control to determine architecting concepts and principles, Ph.D. thesis, Eindhoven University of Technology, Eindhoven, 1998; ISBN: 90-386-0699-4.

[12] L. Bongaerts, J. Wyns, J. Detand, H. van Brussel, P. Valkenaers, Identification of manufacturing holons, in: Proceedings of the European Workshop for Agent-Oriented Systems in Manufacturing, Berlin, 1996.

[13] A. Koestler, The Ghost in the Machine, Arkana Books, London, 1989.

[14] P. Valckenaers, F. Bonneville, H. van Brussel, L. Bongaerts, J. Wyns, Results of the Holonic Control System Benchmark at K.U. Leuven, Leuven, in: Proceedings of Rensselaer's 4th International Conference on Computer Integrated Manufacturing and Automation Technology (CIMAT), Rensselaer's Polytechnic Institute, New York, USA, 1994.

[15] R. Washington, Abstraction planning in real time. Ph.D. thesis, Department of Computer Science, Stanford University, CA, 1994.

[16] L. Bongaerts, L. Monostori, D. McFarlane, B. Kádár, Hierarchy in distributed shop floor control, in: Proceedings of the IMS-EUROPE 1998, the First International Workshop on Intelligent Manufacturing Systems, Ed. Esprit Working group on IMS \& EPFL, Lausanne, 15-17 April 1998, pp. 97-114.

[17] P. Valckenaers, L. Bongaerts, J. Wyns, Planning systems in the next century (II), in: Proceedings of the ICIMS-NOE ASI96, Toulouse, France, Computers in Industry, V34, N2 (1996) 239-245.

[18] M. Schutten, Shop Floor Scheduling with Setup Times efficiency versus lead-time performance. Ph.D. thesis, Faculty of Mechanical Engineering, University of Twente, Enschede, 1996.

[19] H. Cho, A. Derebail, T. Hale, R.A. Wysk, A formal approach to integrating CAPP and SFC, Department of Industrial Engineering, Texas A\&M University, Texas, USA, Transactions of the ASME 116 (1994) 108-116.

[20] S.H. Huang, H.C. Zhang, M.L. Smith, A progressive approach for the integration of process planning and 
scheduling, Department of Industrial Engineering, Texas Technical University, Lubbock, Texas, USA, IIE Transactions 27 (1995) 456-464.

[21] M.M.T. Giebels, H.J.J. Kals, W.H.M. Zijm, Implementation of an engineer-to-order manufacturing planning system (EtoPlan), in: Proceedings of the Flexible Automation and Intelligent Manufacturing 1999 (FAIM'99), Tilburg, The Netherlands, 23-25 June 1999.

[22] M.M.T. Giebels, E.W. Hans, M.P.H. Gerritsen, H.J.J. Kals, Capacity planning for make- or engineer-to-order manufacturing the importance of order classification, in: Proceedings of the 33rd CIRP International Seminar on Manufacturing Systems, Stockholm, Sweden, 5-7 June 2000.

[23] Microsoft Visual $\mathrm{C}^{++}$6.0, http://msdn.microsoft.com/visualc, 1998.

[24] D. Lutters, E. ten Brinke, A.H. Streppel, H.J.J. Kals, Computer aided process planning for sheet metal based on Information Management, in: Proceedings of the 6th International Conference on Sheet Metal (SheMet'98), Enschede, 1998; ISBN: 90-36511135.

[25] M.M.T. Giebels, D. Lutters, H.J.J. Kals, Order information structure in integrated manufacturing planning, in: Proceedings of the 32nd CIRP International Seminar on Manufacturing Systems, Leuven, Belgium, 24-26 May 1999.

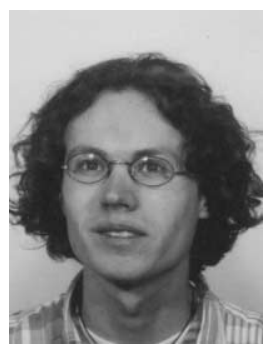

Mark Giebels became Master of Science at the Department of Mechanical Engineering of the University of Twente, Enschede, The Netherlands, in 1995. $\mathrm{He}$ finished his $\mathrm{PhD}$ on flexible manufacturing planning and control systems at the University of Twente in June 2000. This paper provides an overview of the results from his $\mathrm{PhD}$ thesis. He has worked as an assistant professor at the Design, Production and Management group of the University of Twente until April 2001. He is now a researcher at the Siemens Technology-to-Business centre in Berkeley, CA, USA.

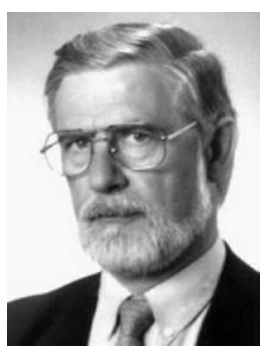

Hubert Kals serves since 1977 as a professor of production engineering and head of the Department of Design, Production and Management of the Faculty of Mechanical Engineering of the University of Twente, Enschede, The Netherlands. Apart from this, he also serves since 1993 as a part-time professor at the Technical University of Delft. Since 1995 , he is the (founding) director of the Centre for Integrated Manufacturing and Development of the University of Twente (CIPV). Since 1998, he is also the (founding) director of The Netherlands Research School for Integrated Manufacturing and Development (IPV). Dr. Kals is a member of the International Institution of Production Research, CIRP, and was its president in 1997/1998. He was, amongst others, awarded and the Frederick W. Taylor Award for Manufacturing Research of the Society of Manufacturing Engineers (SME) of America in 1998.

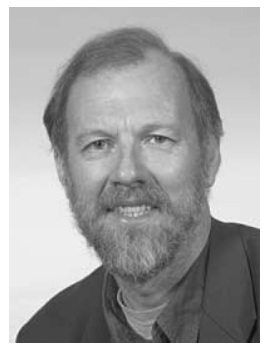

Henk Zijm is the scientific director of the Centre for Telematics and Information Technology (CTIT) of the University of Twente, Enschede, The Netherlands. He is professor and chairman of the Stochastic Operations Research group of the Faculty of Mathematical Sciences, University of Twente, Enschede, The Netherlands. 